\title{
Molecular chemical doping for improvement of performance in organic field effect transistors
}

\begin{abstract}
In this article, we will shortly review molecular doping of organic semiconductors, including polymers, applied to OFETs. The review will cover the main outstanding research achievements reported in the field of organic transistors via doping. Before focusing on these achievements, we will give a brief theoretical background on the organic semiconducting materials, their electronic properties, and organic field - effect transistors as well as main doping fundamentals. We will also discuss some of the reported organic semiconducting materials and doping agents applied for them so far. The article will be concluded with a brief summary and outlook over the doping work done recently and its contributions to the development of organic electronics.
\end{abstract}

Keywords: semiconducting materials, electrical properties, polarity, mobility, stability
Volume 3 Issue 5 - 2019

\author{
Gunel Huseynova \\ Dongguk University, South Korea
}

Correspondence: Gunel Huseynova, Dongguk University, Pildong-ro 30, Jung-gu, Seoul, South Korea, Tel: +82 10 3064-5407, Email bblg7839@gmail.com

Received: June 30, 2019 | Published: September 05, 2019
Abbreviations: OFETs, organic field effect transistors; OLEDs, organic light - emitting diodes; OPVs, organic photovoltaics; LUMO, lowest unoccupied molecular orbital; VB, valence band

\section{Introduction}

The development of organic semiconducting materials paved a path for developing a range of organic devices such as organic field effect transistors (OFETs), organic light - emitting diodes (OLEDs), organic photovoltaics $(\mathrm{OPVs})^{1}$ and so on. There are a lot of advantages of these organic semiconducting materials and devices made based on them over their inorganic counterparts such as unique physical and mechanical properties which in its turn opens opportunities for flexible, light - weight, printable and even paperbased devices. ${ }^{2-5}$ Organic materials are also very easily processed. They offer fabrication methods ranging from conventional thermal evaporation to solution - processing that enables simple fabrication steps such as spin - coating, ${ }^{6}$ drop - casting, ${ }^{7}$ inkjet - printing $^{8}$ and etc. at temperatures as low as room temperature. Also, organic materials are plenty and hundreds of them are synthesized every year. They offer a great range of options for synthesizers. But the main advantage of these organic materials and devices is that they themselves and the processes they go through are not as expensive and complicated as those of their inorganic counterparts. But organic semiconductors suffer from very serious problems such as very poor electrical properties and stability. Although organic semiconductors offer a great range of application fields, these two issues make them fall behind the inorganic semiconductors.

In order to address the abovementioned problems, material scientists and engineers have developed several ways for the last few decades since the organic electronics appeared. Some of these ways have proved themselves to be really straightforward methods for the improvement of the electrical properties and stability of organic semiconducting materials. These methods include doping in the first place, ${ }^{9}$ and then dielectric engineering, ${ }^{10}$ process engineering and so on. Doping has already been demonstrated and applied as the best improvement method for inorganic electronics including silicon technology, for a long time. ${ }^{11}$ The charge carrier concentration, the charge polarity, mobility, stability and other related intrinsic electrical properties can be significantly improved and enhanced several orders of magnitude via doping. ${ }^{12}$ As an example, we can show a very famous organic electron transporting material, $\mathrm{C}_{60}$, intrinsic conductivity of which has been enhanced more than five orders of magnitude by doping. ${ }^{13}$ But the doping technique in organic electronics is very different from that of inorganic electronics. Doping of inorganic materials involves replacement of matrix atoms by the impurity atoms whereas doping of organic materials is nothing more than simple charge transfer process. This review article will mainly focus on the doping, namely molecular chemical doping of organic semiconducting materials, mostly the polymer ones, in the field of OFETs and will go through the main outstanding research achievements reported in the field of organic transistors via doping procedure. Before reviewing these achievements, it will give a brief theoretical background on organic semiconducting materials, their electronic properties and organic field - effect transistors and main doping fundamentals. Then we will discuss the semiconducting materials and doping agents applied for them so far. We will conclude the article with a brief summary and outlook over the doping work done recently and its contributions to the development of organic electronics.

\section{Theoretical background}

\section{Organic semiconductors}

Until the present century silicon - or germanium - based inorganic semiconductors dominated in electronics. These materials have outstanding electrical properties such as high mobility, ${ }^{14}$ high stability ${ }^{15}$ that originate from their single - crystalline structure. ${ }^{16}$ Although inorganic materials themselves are abundant and not so expensive, ${ }^{14}$ the processes involved in their device fabrication methods are very complicated and also very costly. ${ }^{17}$ Besides, inorganic semiconductors are not suitable for flexible, ${ }^{18}$ optoelectronic, ${ }^{19}$ and large - area 
applications. ${ }^{20}$ In addition, with time electronics developed to the extent of downscaling of electronic components such as transistors to nanometer sizes which made it inevitable for starting the searches for alternatives to use instead of these inorganic materials. The searches revealed a new class of materials with semiconducting properties. These materials are known as organic materials that consist mainly of carbon - based molecules. Organic materials demonstrate suitable mechanical and optical properties for modern flexible electronics. In the beginning, the studies of organic semiconductors included mostly single crystal small molecules such as pentacene, anthracene and other acenes. The new epoch has begun in the world of organic electronics when first conductive organic polymer, polyacetylene was dicovered and developed by three great scientists of our age, Alan J. Heeger, Alan G. MacDiarmid and Hideki Shirawa. This discovery, which can also be considered the first doping experience in organic electronics (the scientists doped polyacetilene with chlorine, bromine and iodine vapor via chemical reaction as the result of which the polymer started to exhibit electrical conductivities $10^{9}$ times more than its original (intrinsic) conductivity), changed the whole view about polymers which were considered only insulators until then.
These conductive polymers offer electrical and optical properties similar to those of inorganic semiconductors. In addition, they also possess superior mechanical properties very suitable for flexible electronics. Conductive polymers are also very easy to synthesize. Conductive polymers contain a conjugated backbone through their molecular structure. This structure is held together by very strong and localized sigma bonds and weaker $\pi$-bonds that are the main reason of delocalized $\pi$-electrons along the polymer chain. These $\pi$-electrons make the material conduct the electricity. But unfortunitely, the number of these $\pi$-electrons in these organic polymers is not sufficient for applicable conductivity. Their stability is also one of the main issues to be addressed. For these reasons, doping method is used for adding extra charge carriers (electrons or holes) to the polymer chain in order to enhance its electronic properties and stability. Figure 1 illustrates molecular structures of some representative organic polymers and the generalized energy band scheme for them. While some decades ago polymers were applied only for plastics industry and insulating purposes, being doped, they can be applied in transistors, diodes, solar cells and so on. ${ }^{21}$
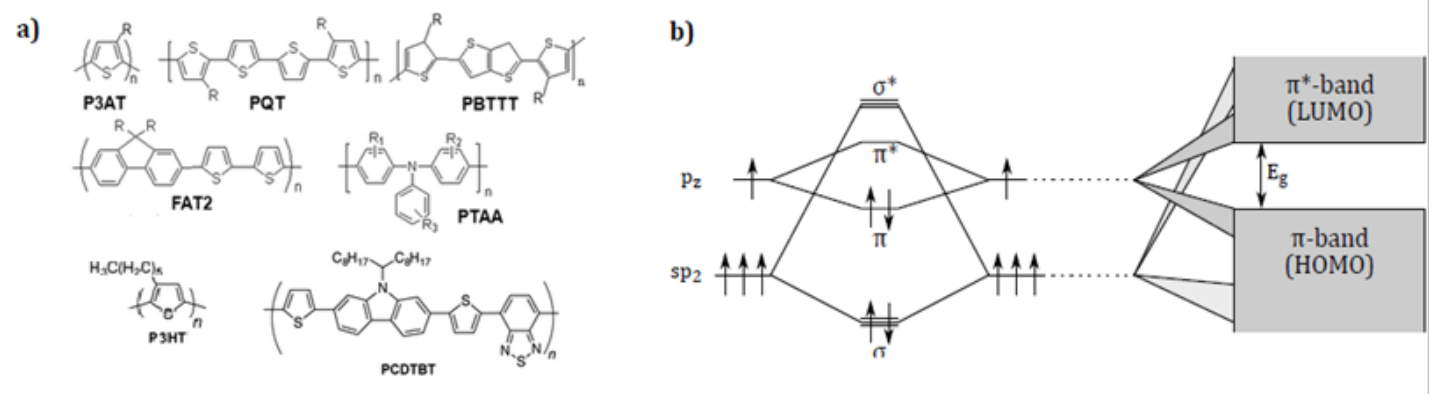

Figure I (A) Molecular structures of some state-of-the art semiconducting polymers; (B) energy band scheme of conjugated polymers.

Charge carrier types in organic semiconducting materials: Organic semiconductors, beside being categorized into two divisions as small molecules and polymers, are also classified as $p$ - type and $\mathrm{n}$ - type semiconductors as their inorganic counterparts. Intrinsically, all semiconducting materials should be electron - transporting, that is, $\mathrm{n}$ - type semiconductors. Neverthelesess, they are classified either as $\mathrm{p}$ - or n-type semiconducting materials. In inorganic electronics, $\mathrm{n}$ - or p-type semiconductor means a semiconducting material heavily doped with electron - abundant (phosphorus, arsenic or other V column chemical element) or electron - defficient (boron, gallium or other III column chemical element) via the way of replacement of the matrix lattice atoms with the dopant atoms. As the conclusion, all inorganic n- or p-type semiconductors are extrinsic semiconductors. For organic semiconductors, the situation is quite different. In order to be classified into p- or n-type semiconductors, they don't necessarily need to be additionaly doped. They are intrinsically either $\mathrm{p}$ - or n-type materials. This can be explained as following. In most organic materials electrons are heavily trapped as the result of very disordered molecular structure and a lot of localized trap states thoughout the polymer backbone. This feature makes them referred to as p-type semiconductors. But still there are some very few organic semiconducting materials in which electrons are not so trapped and can move freely and carry the charge leading to a current flow. The latter makes them n-type semiconductors. Figure 2 illustrates band structures of $\mathrm{n}$ - and $\mathrm{p}$-type semiconductors and chemical structures of representative current $\mathrm{n}$ - and $\mathrm{p}$-type polymer semiconductors. There is also one newly emerged type of organic semiconducting materials which is known as an ambipolar semiconductor. For these materials, hole and electron transport are both characteristic electrical properties. This kind of ambipolar transport is usually observed in narrow band gap organic semiconductor.

Charge transport mechanisms in organic semiconducting materials: As organic polymer semiconductors are bonded mostly via van - der - Waals bonds, the charge transfer in their structure is different from that of the inorganic materials. Charge transfer in these materials happens through the whole molecule rather than inside an individual atom which makes the whole charge transport to be more like charge moving with the lattice deformation around it (charge phonon coupling) which is known as a polaron transport or charge hopping between localized states (charge being sequentially trapped and released) rather than the bandlike transport (charges are promoted to the related energy levels via external excitation) which is mostly encountered in inorganic semiconducting materials. Therefore, in organic electronics, the conduction band (CB) and valence band (VB) are commonly associated with the lowest unoccupied molecular orbital (LUMO) and the highest occupied molecular orbital (HOMO) respectively, of the material of interest. These levels are referred to as the energy levels of the materials and they play very improtant part in organic electronics and the most important part in the doping process of organic materials. The another very important factor is so called Fermi level. For organic semiconductors, the Fermi level can 
be understood as available energy states that can be occupied either by electrons or by holes. The position of this Fermi level is what defines the materials polarity. If it is near the CB (LUMO, respectively), then the material exhibits good electron - transporting properties (n-type), if it is located near the VB (HOMO, respectively), the material is a good hole - transport semiconductor (p-type). For ambipolar semiconductors, this level is considered to be somewhere in between the $\mathrm{CB}$ and VB. Figure 3 illustrates differences in bonding properties of inorganic and organic materials and the position of the Fermi level in different types of organic semiconductors and the scheme of charge transport in organic polymer semiconductors.

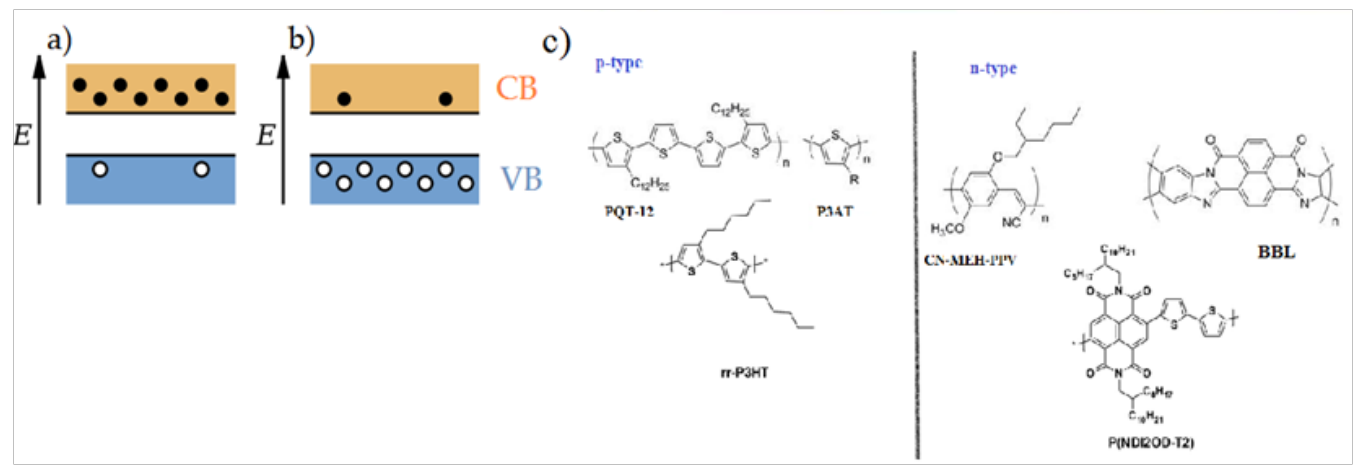

Figure 2 (A) Band structure of an n-type semiconducting material; (B) band structure of a p-type semiconducting material. Dark circles in the conduction band are electrons and light circles in the valence band are holes. In an n-type semiconductor the electrons are the majority charge carriers and holes are the minority charge carriers whereas in a p-type semiconductor the holes are the majority charge carriers and respectively, the electrons are the minority charge carriers; (C) commonly reported $n$ - and $\mathrm{p}$-type polymer semiconducting materials.

a)

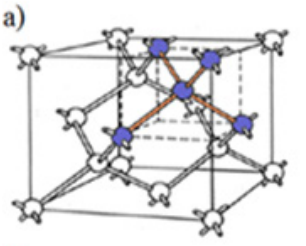

d) i)

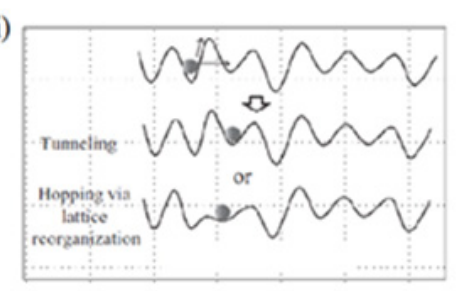

b)

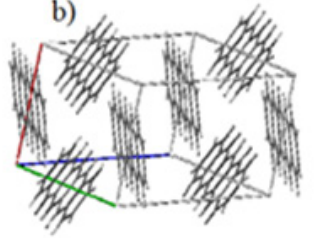

c)

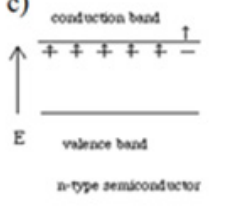

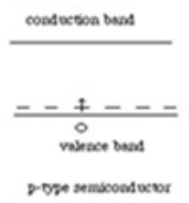

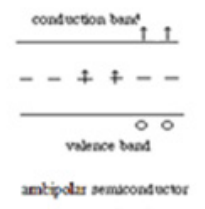

ii)

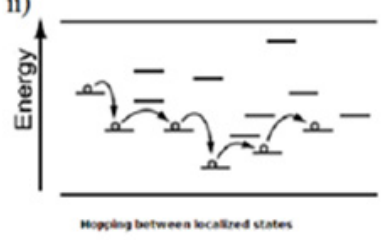

Figure 3 (A) Bonding in inorganic semiconducting materials - covalent (as in Si) or ionic bonds (as in GaAs); (B) bonding in organic semiconducting materials Van - der - Waals bonding (bonding between covalently bonded molecules); (C) the Fermi level position in n-, p-type and ambipolar semiconductors; (D) charge transport mechanism in organic polymer semiconductors - (i) the possible tunneling and hopping via polaron transport and (ii) charge trapping and releasing (hopping) through the localized energy states.

\section{Organic field - effect transistors}

Organic Field - Effect Transistors (OFETs) are a kind of field effect transistors (FETs) that uses organic semiconducting material in its active channel. They are also known as Organic Thin-Film Transistors (OTFTs) as both the active and insulating layers comprise very thin films in these transistors. The first OFET was fabricated by Koezuka and his colleagues in 1987 based on thiophene conducting polymer material. The device structure is the same as in Metal Oxide Semiconductor Field-Effect Transistors (MOSFETs), another kind of FETs that consists of three main terminals via biasing one of which (Gate) the current (charge carrier density) can be controlled between the other two (Source and Drain). As the main factor of these transistors is the use of organic materials as an active layer, the thin insulating layer that separates the Gate terminal of the transistor form the Source and Drain terminals and the conductive channel between them (gate dielectric) can be selected from both organic and inorganic materials. Nevertheless, nowadays scientists are trying to use more and more organic materials for the insulating layer, too, in order to introduce all - organic or all - polymer based transistors. The main output of this input gate voltage in these devices is the drain current as in other kinds of transistors. The drain current and the other main parameters of OFETs such as charge carrier mobility, threshold voltage, on/off current ratio, can be extracted both in linear and saturation regime of 
the device. The below given equations represent these calculations for both regimes accordingly:

$$
\begin{aligned}
& I_{D}=\frac{w}{L} C_{i} \mu\left(\mathrm{V}_{G}-\mathrm{V}_{T}\right) \mathrm{V}_{D}-\text { linear regime; } \\
& I_{D}=\frac{w}{2 L} C_{i} \mu\left(\mathrm{V}_{G}-\mathrm{V}_{T}\right)^{2}-\text { saturation regime; }
\end{aligned}
$$

where

$I_{D}-$ drain current $(A)$;

$\mathrm{W}$ - channel width ( $\mathrm{mm}$ or $\mu \mathrm{m})$;

$\mathrm{L}$ - channel length (usually in $\mu \mathrm{m}$ );

$\mathrm{C}_{\mathrm{i}}-$ the gate dielectric capacitance per unit area $\left(\mathrm{F} / \mathrm{cm}^{2}\right)$;

$\mu$ - charge carrier mobility $\left(\mathrm{cm}^{2} / \mathrm{Vs}\right)$;
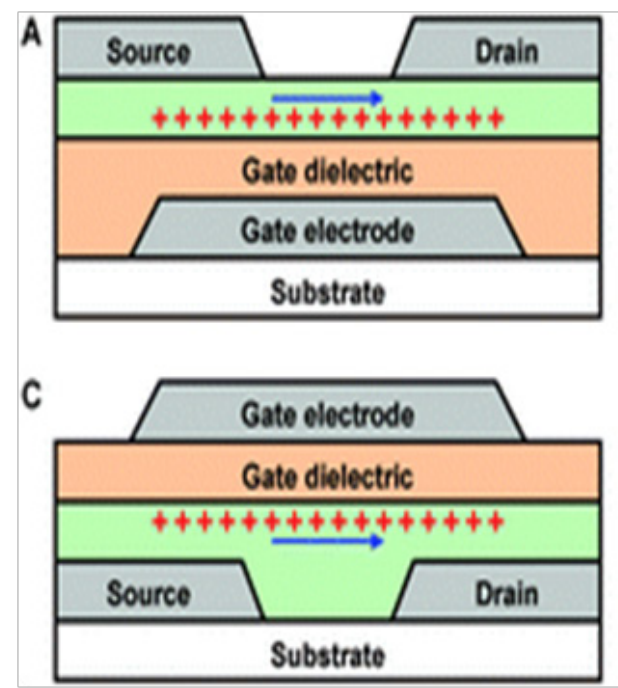

Figure 4 (A) BGTC; (B) BGBC; (C) TGBC; and (D) TGTC.

\section{Doping fundamentals}

Doping is understood as introduction of impurities into the host materials for the purposes of improvement and enhancement of their properties of interest. In inorganic electronics, doping is a procedure where a dopant atom replaces a matrix atom in its lattice and this way, provides it with an extra electron or hole. But in organic electronics, doping is totally different. Here there is no replacement of matrix atoms by impurity atoms. This process should be understood as a simple charge transfer process between two materials which are referred to as donor or acceptors. Depending on the type of doping and energy levels of materials used, the same dopant can be used both as a donor or an acceptor for different host materials. There are four common ways used for doping organic polymers. They are chemical doping which is the first doping mechanism ever used, electrochemical doping, interfacial doping and photo - induced doping. In chemical doping, organic matrix molecule and the doping agent can be either blended in a solution or thermally co-evaporated. Although electrochemical doping is not common for OFETs, there have been some reports on the OFETs based on electrolyte - gate dielectric where possibly
$\mathrm{V}_{\mathrm{G}}$ - applied gate voltage $(\mathrm{V})$;

$\mathrm{V}_{\mathrm{T}}$ - threshold voltage $(\mathrm{V})$;

$\mathrm{V}_{\mathrm{D}}$ - drain voltage $(\mathrm{V})$.

Depending on the position of the source and drain terminals with respect to the active layer of the device, OFETs are usually configured in two main device structures: top contact (TC) - when the source and drain electrodes are deposited on the top of the organic material and bottom contact (BC) - when the source and drain electrodes are deposited under the organic semiconductor. Depending on the position of the gate terminal and considering the above - mentioned possible two device configurations, OFETs are designed in four main geometries: top - gate top - contact (TGTC), bottom - gate bottom - contact (BGBsC) - the coplanar geometries and top - gate bottom contact (TGBC), bottom gate top contact (BGTC) - the staggered geometries. Figure 4 illustrates the common four OFET device geometries.
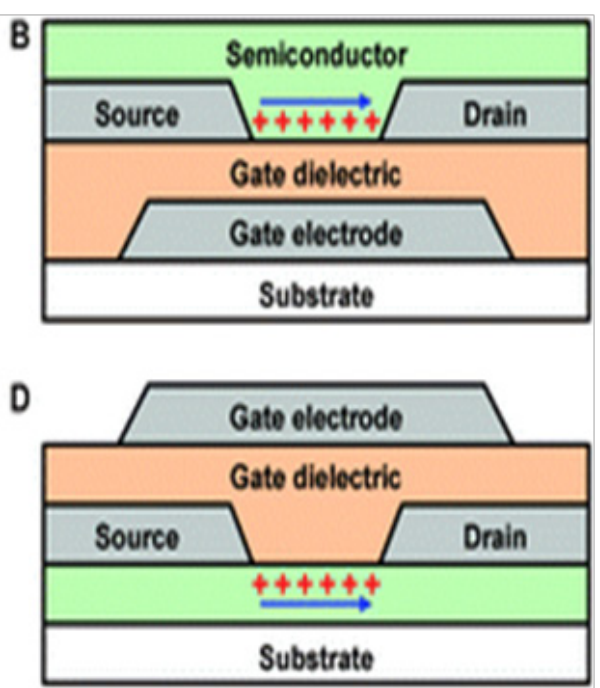

this kind of doping process takes place. Interfacial doping involves injection of relative charge carriers (electrons or holes) depending on the polarity of the gate voltage, from metal contacts into the organic semiconductor. And finally, the last doping mechanism - photo induced doping, may be considered the most complicated and time - demanding method for doping as it involves several complicated steps such as thermal co - evaporation, extra light excitement in order to excite the electron to jump to a level much higher than its binding energy, then yet another step like hydride or another ion transfer to the dopant molecule, is needed to prevent this electron to fall back again to its former energetic state as it is lower than its current state The doping effect becomes permanent by passivation of the positive charge (hole) left in the former electron place by a hydride transfer. To give a little more insight for this photo - induced doping, Ref. 22 is provided (see Scheme 1 on page 6). ${ }^{13}$

\section{P-type doping}

As it was already mentioned, in organic electronics, the energy levels, namely the HOMO and LUMO levels of both the matrix 
molecules and the dopant molecules play the essential part in the doping process. In case of p-type doping, the HOMO level of the organic matrix molecule should lie higher than the HOMO or LUMO levels of the doping agent. In this case, the dopant molecule is referred to as an acceptor while the host molecule is the donor.

\section{N-type doping}

In n-type doping, the dopant molecule plays the role of the donor and its HOMO level should be higher than the LUMO level of the matrix molecule which is in this case the acceptor. Figure 5 illustrates the $\mathrm{p}$ - and n-type doping process in organic semiconducting materials.

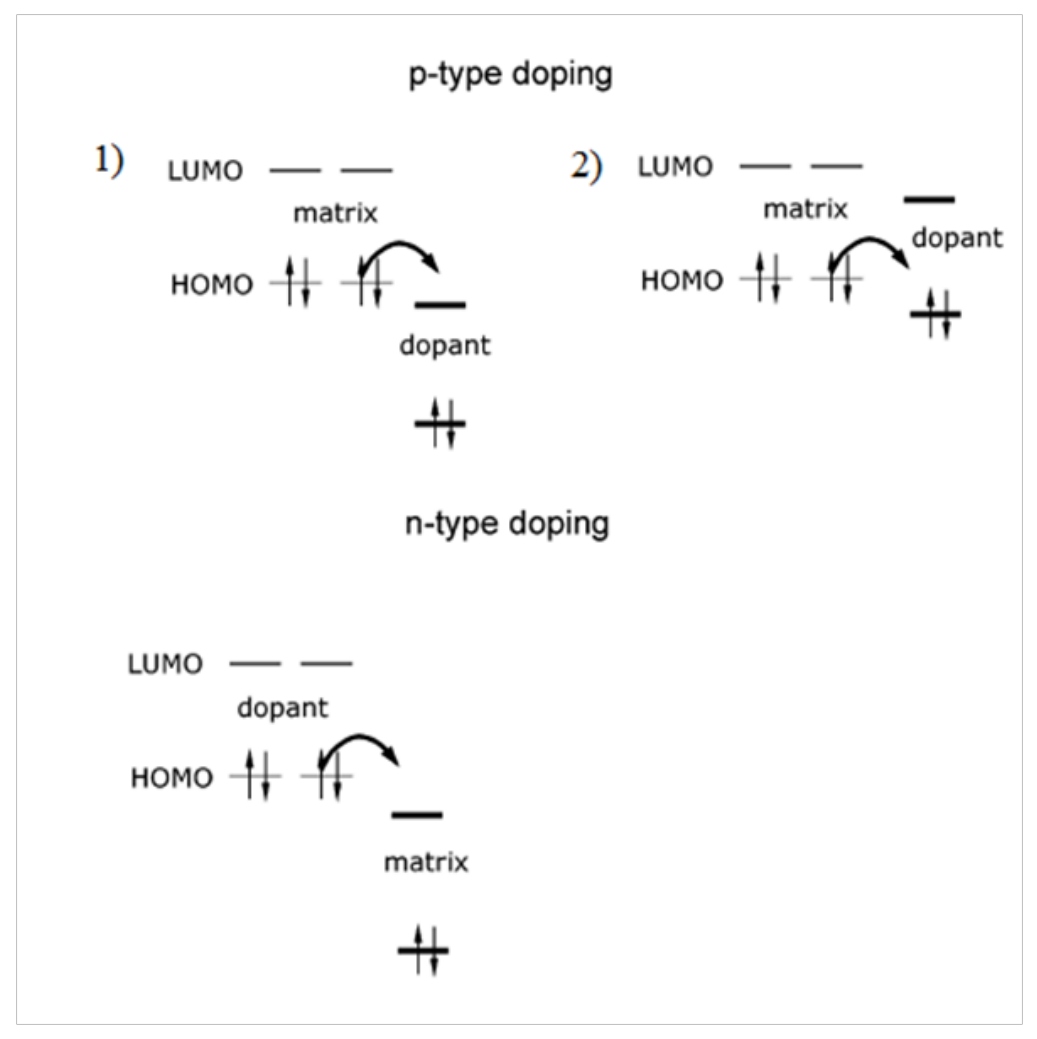

Figure 5 Schematic illustration of the molecular $\mathrm{p}$ - type and $\mathrm{n}$ - type doping mechanisms.

\section{Materials}

\section{Matrices}

Doping has proved itself to be the best way for the improvement of electrical properties as well as the stability of the OFET devices based on polymeric semiconductors. Key materials used as host matrices in doping studies include a wide range of organic structures starting from small molecules such as pentacene up to great number of polymers such as P3HT, N2200, DPP - based polymers and so on. As the main objective of this review article is the molecular doping of OFETs based on polymer semiconducting materials, we will mainly focus on macro molecules. In this case, doping is mainly a process of blending the host and impurity molecules in the same or different solvents. We will also give a brief view for doping processes of OFETs using small molecules in their active channel in the chapter about doping with organic dyes.

\section{Dopants}

For the doping purposes different kinds of doping agents have been used since the beginning of doping studies. These dopants include both organic and inorganic structures. The most frequently used materials for $\mathrm{p}$ - channel are doping tetrafluoro-tetracyanoquinodimethane (F4-TCNQ) which is a very strong electron acceptor, molybdenum-, vanadium oxides and so on. As for n-type doping, it is much more challenging to find reliable doping agents. Nevertheless, inorganic materials involving alkali metals, alkali metal salts such as cesium fluoride or cesium carbonate, as well as organic dopants such as cobaltocene have been successfully used for this purpose.

\section{Doping with inorganic and organic materials}

Chuan et al., ${ }^{9}$ have demonstrated significantly improved mobility, on/off current ratio, crystallinity, injection and reliability in n-type polymer P(NDI2OD-T2) based OFETs via molecular doping. The $\mathrm{P}(\mathrm{NDI} 2 \mathrm{OD}-\mathrm{T} 2)$, also known as N2200, is a conjugated electron transport polymer with a very low lying LUMO level (around $-4.0 \mathrm{eV}$ ) which makes it very suitable for n-type doping. By using two different classes of organic and inorganic dopants, namely, cobaltocene $\left(\mathrm{CoCp}_{2}\right)$ and cesium fluoride $(\mathrm{CsF})$, respectively, they were able to enhance the FET mobility twice and decrease the threshold voltage almost four times. Both the dopants resulted in an increase in carrier concentration which in its turn smoothed the path for charge carriers as the result of filled traps by extra carriers. The Fermi level was effectively shifted towards the LUMO level of the host material. Remarkably, both dopants exhibited the same tendency in mobility change. Mobility continuously increased by increasing the dopant concentration as the significant number of trap states were 
filled and extra charge carriers were introduced. Nevertheless, after some critical point which was $0.5 \mathrm{wt} \%$ dopant concentration in the said research, additional concentration did not improve the mobility but decreased it even below the intrinsic value. This phenomenon was attributed to the molecular structure disturbance because of the introducing of too many impurity carriers which in such a case play also the role of additional trap sites. ${ }^{9}$

Another very impressive work was done by Dongyyoon Khim and his colleagues in 2014. He demonstrated an effective way for controlling polarity of charge transport in OFETs by selective molecular doping. Via blending a small amount of two different molecular dopants, F4-TCNQ for $\mathrm{p}$ - channel doping and CsF for $\mathrm{n}$ - channel doping he could selectively tune the operational polarity of intrinsically ambipolar methanofullerene $[6,6]$-phenyl- $\mathrm{C}_{61}$-butyric acid methyl ester (PCBM) from ambipolar transport to unipolar, either electron - or hole - transport in OFETs. This method is very effective for complementary integrated circuits based on organic semiconductors. By this doping process the same organic material can be doped both ways to comprise a $\mathrm{p}-\mathrm{n}$ junction just as in silicon electronics. ${ }^{22}$ Yuan Zhang et al., ${ }^{23}$ achieved a trap - free space charge limited electron transport in a conjugated polymer poly[2methoxy-5-(2-ethylhexyloxy)-1,4-phenylenevinylene] (MEH-PPV) which is a $\mathrm{p}$ - type semiconductor as the electrons are strongly trapped in its backbone, by doping it with an $\mathrm{n}$ - channel dopant decamethylcobaltocene (DMC). The deep traps in the polymer's structure were effectively deactivated (filled) by the dopant - induced carriers. ${ }^{23}$ Another group of researchers. A Nollau ${ }^{11}$ and his coworkers, reported a controlled $\mathrm{n}$ - type doping of organic semiconductors. In their research they applied doping by co - sublimation of two semiconducting materials one of which is NTCDA, acting as the host structure and being a strong electron acceptor and electron transport material. As the dopant, bis(ethylenedithio)-tetrathiaflvalene (BEDTTTF) charge - transfer salt that is itself a semiconducting material, was used. ${ }^{11}$ Yabinq Qi et al., ${ }^{24}$ reported air - stable $\mathrm{n}$ - type doping agents, $\left[\mathrm{RhCp}_{2}\right]_{2}$ and $[\mathrm{Cp} * \mathrm{Ru}(\mathrm{TEB})]_{2}$ for polymeric organic semiconductors such as N2200 and small molecule organic semiconductors such as TIPS-pentacene, current density, conductivity and stability of which increased several orders of magnitude thanks to the doping. ${ }^{24}$ Calvin K Chan and his colleagues have once again confirmed that a strong reducing agent, $\mathrm{CoCp}_{2}$ is a very good molecular dopant for $\mathrm{n}$ - channel doping of organic host materials. The doping process they used is a little different from the conventional solution or co evaporation doping of organic materials. $\mathrm{CoCp}_{2}$ was incorporated as a doping layer grown by cobaltocene vapor with host material film that was thermally evaporated. This type of doping can be referred to as a gas - phase or vapor doping. Current density was significantly increased by this doping method. ${ }^{25}$ Selina Olthof et al., ${ }^{26}$ achieved a controlled passivation of acceptor tail states that act as charge traps, in fullerene $\mathrm{C}_{60}$ by ultralow molecular $n$-doping. By successfully filling the traps with very small amount of dopant material, they were able to obtain increased conductivity, mobility, and decreased activation energy. They also effectively controlled Fermi level position and work function of the material. ${ }^{26}$

\section{Doping with cationic dyes}

Another class of doping materials that have been used as molecular $\mathrm{n}$ - channel dopants by some research groups $\mathrm{s}^{27,28}$ covers cationic dyes which are basically organic salts. The doping process involving these organic dyes has been photo - induced doping so far that has already been discussed in the introduction part of doping fundamentals. Both the host and the doping molecules are thermally co - evaporated to form a uniform film. During the co - evaporation process, the dopant organic molecules undergo oxidation and form their leuco bases which act as the basic doping agents. The host materials used for the study have included non - polymeric organic semiconductors such as NTCDA, $\mathrm{F}_{16} \mathrm{ZnPc}$, TCNQ, F4-TCNQ, and $\mathrm{C}_{60}$ which are very strong electron acceptors. The cationic dyes used as doping agents include Pyronin B (PyB), Crystal Violet (CV), Acridine Orange (AO), Malachite Green (MG), and their leuco - bases. ${ }^{13,27-30}$

\section{Summary and outlook}

This review article has discussed the molecular doping of OFETs by the means of co - solvent blending and co - evaporation. For doping of organic materials both organic and inorganic materials can be employed. The doping mechanism used for organic electronics is totally different from that of inorganic electronics. The structural and electrical properties as well as stability of organic semiconducting materials including both small and macro molecules, can be remarkably improved by doping techniques. The resultant materials with enhanced properties can be applied to a wide range of electronic devices such as OLEDs, OFETs, CMOS - like integrated circuits, etc. However, there are still some points that should be cleared and understood in doping of organic semiconductors. In addition, even though doping improves and enhances the device performance, organic electronics is still falling behind the inorganic electronics. Therefore, new, powerful and reliable dopants for organic materials have to be developed.

\section{Acknowledgements}

None.

\section{Funding details}

None.

\section{Conflict of interest}

Authors declare that there is no conflict of interest.

\section{References}

1. Wang C, Dong H, Jiang L, et al. Organic semiconductor crystals. Chem Soc Rev. 2018;47:422-500.

2. Christian Kunkel, Christoph Schober, Johannes T Margraf, et al. Finding the Right Bricks for Molecular Legos: A Data Mining Approach to Organic Semiconductor Design. Chemistry of Materials. 2019;31(3):369-378.

3. Erfan Mohammadi, Chuankai Zhao, Yifei Meng, et al. Dynamictemplate-directed multiscale assembly for large-area coating of highlyaligned conjugated polymer thin films. Nature Communications. 2017;8.

4. Gupta SK, Jha P, Singh A, et al. Flexible organic semiconductor thin films. J Mater Chem C. 2015(3):8468-8479.

5. Irimia-Vladu M, Glowacki ED, Voss G, et al. Green and biodegradable electronics. Materials today. 2012;15(7-8):340-346.

6. Chou KW, Khan HU, Niazi MR, et al. Late Stage Crystallization and Healing During Spin-Coating Enhance Carrier Transport in SmallMolecule Organic Semiconductors. J Mater Chem C. 2014;2:5681-5689.

7. Arnaud D, Pandey RK, Miyajima S, et al. Fabrication of Large-scale Drop-cast Films of $\pi$-conjugated Polymers with Floating-film Transfer Method. Trans Mat Res Soc Japan. 2013;38(2):305-308. 
8. Cho SY, Ko JM, Lim J, et al. Inkjet-Printed Organic Thin Film Transistors Based on TIPS Pentacene with Insulating Polymers. J Mater Chem C. 2013;1:914-923.

9. Chuan Liu, Junhyuk Jang, Hyo-Jung Kim, et al. Effect of Doping Concentration on Microstructure of Conjugated Polymers and Characteristics in N-Type Polymer Field-Effect Transistors. Adv Func Mat. 2015;25:758-767.

10. Baeg KJ, Khim D, Jung SW, et al. Remarkable Enhancement of Hole Transport in Top-Gated N-Type Polymer Field-Effect Transistors by a High-k Dielectric for Ambipolar Electronic Circuit. Adv Mater. 2012;24:5433-5439.

11. Nollau A, Pfeiffer M, Fritz T, et al. Controlled n-type doping of a molecular organic semiconductor: Naphthalenetetracarboxylic dianhydride (NTCDA) doped with bis(ethylenedithio)-tetrathiafulvalene (BEDT-TTF). J Appl Phys. 2000;87(9):4340.

12. Lüssem, Riede M, Leo K. Doping of Organic Semiconductors. Phy Status Solidi A. 2013;210(1):9-43.

13. Fenghong Li, Ansgar Werner, Martin Pfeiffer, et al. Leuco Crystal Violet as a Dopant for n-Doping of Organic Thin Films of Fullerene C60. J Phys Chem B. 2004;108:17076-17082.

14. Ameen S, Akhtar MS, Shin HS, et al. Chapter Five - Charge-Transporting Materials for Perovskite Solar Cells. Advances in Inorganic Chemistry. 2018;72:185-246.

15. Eslamian M. Inorganic and Organic Solution-Processed Thin Film Devices. Nano-Micro Lett. 2017;9:3.

16. Ma Z, Qin G. Semiconductor Nanomaterials for Flexible Technologies. 2010:67-104.

17. Langa F, Nierengarten JF. Fullerenes: Principles and Applications. RCS Publishing; 2007

18. Yu Oshima, Atsutomo Nakamura, Katsuyuki Matsunaga. Extraordinary plasticity of an inorganic semiconductor in darkness. Science. 2018;360(6390):772.

19. Cacialli F. Organic Semiconductors for the New Millennium, Philosophical Transactions: Mathematical, Physical and Engineering Sciences Vol. 358, No. 1765, Science into the Next Millenium: Young Scientists Give Their Visions of the Future: II. Mathematics, Physics and Engineering. 2000:173-192.
20. Kitai A. Organic Semiconductors, OLEDs and Solar Cells, Principles of Solar Cells, LEDs and Diodes: The Role of the PN Junction. 2011: 287.

21. Kumar R, Singh S, Yadav BC. Conducting Polymers: Synthesis, Properties and Applications. International Advanced Research Journal in Science, Engineering and Technology. 2015;2(11).

22. Dongyoon Khim, Kang-Jun Baeg, Mario Caironi, et al. Control of Ambipolar and Unipolar Transport in Organic Transistors by Selective Inkjet-Printed Chemical Doping for High Performance Complementary Circuits. Ad Func Mat. 2014;24:6252-6261.

23. Yuan Zhang, Bert de Boer, Paul WM. Trap-free electron transport in poly(p-phenylene vinylene) by deactivation of traps with n-type doping. Phys Rev B. 2010;81:085201.

24. Yabing Qi, Swagat K Mohapatra, Sang Bok Kim, et al., Solution doping of organic semiconductors using air-stable n-dopants. Appl Phys Lett. 2012;100:083305

25. Calvin K Chan, Fabrice Amy, Qin Zhang, et al. N-type doping of an electron-transport material by controlled gas-phase incorporation of cobaltocene. Chem Phys Lett. 2006;431:67-71.

26. Selina Olthof, Shafigh Mehraeen, Swagat K Mohapatra, et al. Ultralow Doping in Organic Semiconductors: Evidence of Trap Filling. PRL. 2012;109:176601.

27. Calvin K Chan, Eung-Gun Kim, Jean-Luc Bredas, et al. Molecular n-Type Doping of 1,4,5,8-Naphthalene Tetracarboxylic Dianhydride by Pyronin B Studied Using Direct and Inverse Photoelectron Spectroscopies†. Adv Funct Mater. 2006;16:831-837.

28. Ansgar Werner, Fenghong Li, Kentaro Harada, et al. n-Type Doping of Organic Thin Films Using Cationic Dyes†. Adv Funct Mater. 2004;14(3): $255-260$.

29. Fenghong Li, Naoki Hayashi, Kazuhiko Seki, et al. Acridine orange base as a dopant for $\mathrm{n}$ doping of $\mathrm{C}_{60}$ thin films. J Appl Phys. 2006; 100:023716.

30. Werner AG, Li F, Harada K, et al. Pyronin B as a donor for n-type doping of organic thin films. Appl Phys Lett. 2003;82(25):4495. 